\title{
DUST GRAIN ORIENTATION IN THE INTERSTELLAR MAGNETIC FIELD
}

\author{
A.Z. DOLGINOV \\ A.F. Ioffe Physical-Technical Institute \\ Academy of Sciences \\ Politechnical str. 26 \\ 194021 Leningrad \\ USSR
}

ABSTRACT. It is shown that the orientation of the dust grain body relative to its angular momentum $J$ by the Barnett relaxation effect and the orientation of $\mathrm{J}$ relative to the interstellar magnetic field, by action of anisotropic gaseous fluxes, which always exist in space, is more effective than the orientation of the Davis-Greenstein mechanism. The linear interstellar polarization, which arises by scattering of radiation on oriented grains, is directed along the galactic magnetic field.

Interstellar polarization is due to the scattering of stellar light at nonspherical dust grains oriented relative to the interstellar magnetic field. The most popular mechanism of orientation is the paramagnetic relaxation considered by Davis and Greenstein (1951). However, it meets many difficulties, and first of all the very long time of orientation $\left(10^{13}-10^{14} \mathrm{~s}\right)$ for non-superparamagnetic grains. The alternative possibility is the orientation in anisotropic gaseous fluxes (Gold, 1952; Dolginov and Mytrophanov, 1976) which exist in all gaseous dust clouds. In all cases the predominant direction of the grain short axis is the field direction because the grain possesses a magnetic moment due to the Barnett effect (Dolginov and Mytrophanov, 1976; Purcell, 1979). This effect leads also to a relaxation process which results in an alignment of the angular momentum $J$ relative to the grain axis in a short time of the order of a few years (Purcell, 1979). So one can consider the orientation of $\mathrm{J}$ relative to the grain body from the very beginning. Using results obtained in Dolginov et al. (1979) we get the distribution function $W(\theta)$ of the angle $\theta$ between $J$ and $B$

$$
W(\theta)=\frac{A}{2}\left[\frac{A+S}{\left(A+S \cos ^{2} \theta\right)^{3}}\right]^{1 / 2}
$$

$A=1$ for prolate and $A=\left[\left(a^{2}+b^{2}\right) / 2 b^{2}\right]<1$ for oblate grains. For the sake of simplicity we consider here only the case of axisymmetric fluxes and axisymmetric grains. $\vec{a}$ is the grain symmetry semi-axis and $\vec{b}$ is perpendicular to it. $S=\left[3 \cos ^{2} \theta-1\right]\left[2 \sin ^{2} \theta\right]^{-1}$ determines the anisotropy of the gaseous flux. $\theta$ is the angle between the flux axis and $B_{\text {out }}$. In an isotropic case and also for $\theta=55^{\circ}$ or $125^{\circ}, \mathrm{S}=0$. If $\theta\left\langle 55^{\circ}\right.$ or $\left.\theta\right\rangle$ 
$125^{\circ}, \mathrm{S}>0$ and if $55^{\circ}<\theta<125^{\circ}, \mathrm{S}<0$. The interstellar polarization depends on averaged values $W_{2}=\left\langle\cos ^{2} \theta_{0}\right\rangle$ and $W_{4}=\left\langle\cos ^{4} \theta_{0}\right\rangle$ where $\theta_{0}$ is the angle between $B_{\text {out }}$ and $\vec{a}$. For the oblate grains

$$
\begin{array}{ll}
W_{2}=\frac{A}{2}\left\{\frac{1}{2} \sqrt{\frac{A+S}{S}} \ln \mid \frac{\sqrt{A+S}+\sqrt{S}}{\sqrt{A+S}-\sqrt{S}}-1\right\} & S>0 \\
W_{2}=\frac{A}{|S|}\left\{1-\sqrt{\frac{A+S}{|S|}} \arcsin \sqrt{\frac{|S|}{A}}\right\} & S<0
\end{array}
$$

For extremely oblate grains $(A \rightarrow-12)$, which are situated in a plane where the flux is directed perpendicular to the field $(S \rightarrow-1 / 2), W_{2}=1$ is maximum. For the prolate grains

$$
\begin{array}{ll}
W_{2}=\frac{1}{2} S\left\{S+\sqrt{\frac{1+S}{4 S}} \ln \mid \frac{\sqrt{1+S}+\sqrt{S}}{\sqrt{1+S}-\sqrt{S}}-1\right\} & S>0 \\
W_{2}=\frac{1}{2|S|}\left\{|S|+\sqrt{\frac{1+S}{|S|}} \arcsin \sqrt{|S|}-1\right\} & S<0
\end{array}
$$

If $\mathbf{S}<0$, the grain minor axis is directed along the field. For gaseous fluxes which are randomly directed relative to the large-scale interstellar field the case $S<0$ is predominant, because the contribution of $55^{\circ}<\theta$ $\left\langle 125^{\circ}\right.$ is larger by a factor 1.38 than that of $\theta\left\langle 55^{\circ}\right.$ and $\theta>125^{\circ}$ (Dolginov et al., 1979), i.e. the average direction of interstellar polarization coincides with the average field direction in agreement with observations. The orientation time $t_{g}$ in the flux can be defined as the time necessary to get the directed component of $\mathrm{J}$ much larger than its random component $\mathrm{J}_{\mathrm{ran}}$. Estimates (Dolginov et al., 1979) give $\mathrm{t}_{\mathrm{g}} \approx\left\langle\mathrm{J}_{\mathrm{ran}}^{2}\right\rangle$ $\left[4 a b^{3} N_{a} m_{a}^{2} v_{a}^{3}\right]^{-1}$, where $m_{a}$ is the mass, $N_{a}$ the number density, $v_{a}$ the directed velocity of the gas particles relative to the grain. $v_{a} \neq 0$ and $10^{5}-10^{6} \mathrm{~cm} / \mathrm{s}$ because the time necessary for the grain to acquire the gas velocity is very large. $\left\langle\mathrm{J}_{\mathrm{ran}}^{2}\right\rangle \approx \mathrm{I}_{\mathrm{g}} \mathrm{k} \mathrm{T}_{\mathrm{gas}}$ where $\mathrm{I}_{\mathrm{g}}$ is the grain momentum of inertia. Taking into account the possibility of $\mathrm{H}_{2}$ recombination on the grain surface, one must take for $\mathrm{T}_{\text {gas }}$ not the kinetic temperature but the temperature $22.10^{3} \mathrm{~K}$, corresponding to the recoil energy of $\mathrm{H}_{2}$ (Purcell, 1979). The orientation time $\mathrm{t}_{\mathrm{g}}$ in the gaseous dust clouds, due to the action of fluxes, is at least an order of magnitude shorter than that for the Davis-Greenstein mechanism.

\section{References}

Davis, J. and Greenstein, J. (1951) Astrophys. J. 114, 87.

Dolginov, A.Z. and Mytrophanov, I.G. (1976) Astrophys. Space Sci. 43, 2.

Dolginov, A.Z., Gnedin, Yu.N. and Silant'ev, N.A. (1979) "Propagation and Polarization of Radiation in Cosmic Media", Nauka, Moscow.

Gold, T. (1952) Monthly Notices Roy. Astron. Soc. 112, 215.

Purcell, E. (1979) Astrophys. J. 231, 404. 
CUGNON: Superparamagnetism is not ferromagnetism: it can preserve the dielectric character of the grains (small metallic inclusions). The dielectric character of the grain is not really shown by the comparison between circular and linear polarization.

DOLGINOV: I did not say that the superparamagnetism is identical with ferromagnetism. The correlation between the linear and circular polarization curves is the same as for dielectric. Of course, it may be the same for special cases of nondielectric substances.

CUGNON: Did you take into account the fluctuating character of the Barnett relaxation torque? These fluctuations are governed by the temperature of the grain, so that the degree of alignment can be reduced like in $D-G$ mechanism.

DOLGINOV: The Barnett magnetic moment depends on the magnetic susceptibility and hence on the grain temperature. But in all cases it is directed along the angular velocity, and its position relative to the field does not depend on the temperature fluctuations. Besides, the fluctuations are smaller than the average temperature and are unimportant.

SHUKUROV: Interstellar gas is in the state of constant, quasi-uniform turbulent motions at scales considerably larger than all relevant scales of your theory. How then would you explain the absence of polarization in some regions?

DOLGINOV: The degree of polarization depends on the angle between the local field lines and the gaseous flux direction. So, in some regions the orientation is not visible even if there are both field and flux.

BECK: Our radio observations indicate that the magnetic field is mainly random in the spiral arms of Sc galaxies where the dust is concentrated. This gives a natural explanation why Dr. Scarrott does not detect optical polarization in the dust lanes of late-type galaxies. 